\title{
$\mathbf{J}|\mathbf{A}| \mathbf{C} \mid \mathbf{S}$ \\ $\overline{\text { COMMUNICATIONS }}$
}

Published on Web 08/10/2002

\section{Traveling Fronts of Copper Deposition}

\author{
Stephanie Thouvenel-Romans, Konstantin Agladze, and Oliver Steinbock* \\ Department of Chemistry and Biochemistry, Florida State University, Tallahassee, Florida 32306-4390
}

Received May 8, 2002

Many chemical and biological systems sustain propagating fronts and complex modes of spatio-temporal pattern formation. ${ }^{1}$ Important examples include concentration waves in autocatalytic reactiondiffusion systems, such as the Belousov-Zhabotinsky reaction ${ }^{2}$ and self-replicating RNA populations, ${ }^{3}$ calcium waves in single cells, ${ }^{4}$ and traveling action potentials in neuronal and cardiac tissue. ${ }^{5}$ Propagating reaction zones have been utilized for the efficient synthesis of hydrogels, ceramics, and superconductors as well as in ring-opening metathesis polymerization. ${ }^{6}$ Also, in electrochemistry these macroscopic wave structures are subject to an increasing number of studies that typically aim to explain the dynamics of dissolving or catalytic electrode surfaces. ${ }^{7}$ Here, we present experimental evidence for propagating fronts that mediate the electroless deposition of copper on passivated steel. Under appropriate reaction conditions, these electrodeposition fronts propagate with constant speeds that fall in the range from $0.5-5 \mathrm{~mm} / \mathrm{s}$. The phenomenon can be observed with the unaided eye, and the front velocity is essentially independent from the copper(II) concentration in the electrolyte, which indicates that the propagation is governed by the features of the thin, passivating iron oxide film.

The classic example for pattern formation in electrodeposition processes is the creation of fractal-shaped metals via diffusionlimited aggregation. ${ }^{8}$ Moreover, traveling fronts and even rotating spiral waves have been observed in the electro-co-deposition of silver-indium and silver-antimony alloys. ${ }^{9}$ Our experiments employ passivated steel samples that are protected by a $10-100 \AA$ thick layer of iron oxide $\left(\mathrm{Fe}_{2} \mathrm{O}_{3}\right.$ and $\left.\mathrm{Fe}_{3} \mathrm{O}_{4}\right)$. This surface film and its stability have been investigated in numerous studies. ${ }^{10}$ Although widely forgotten, a patent from 1913 also suggests nitric acid passivation as a possible strategy to improve the copper plating of steel substrates. ${ }^{11}$ However, today's metal plating techniques are usually based on the alkaline copper-cyanide bath and the sulfuricacid copper bath. ${ }^{12}$

Experiments are carried out on low carbon steel (Mc MasterCarr-C1018; C, 0.15-0.20\%; Mn, 0.60-0.90\%; P, <0.040\%; S, $<0.050 \%)$. The metal plates $\left(10.2 \times 5.0 \mathrm{~cm}^{2}\right)$ are passivated in nitric acid solution (11.5 M; Fisher) prior to the plating experiment. In this procedure, the samples are kept in the nitric acid solution at $0{ }^{\circ} \mathrm{C}$ for $20 \mathrm{~min}$, then washed with distilled water and quickly dried with acetone to prevent corrosion. Aqueous $\mathrm{CuSO}_{4}$ (Fisher) solutions with or without added $\mathrm{Na}_{2} \mathrm{SO}_{4}, \mathrm{NaOH}$, or $\mathrm{H}_{2} \mathrm{SO}_{4}$ serve as the plating bath. The plating process is carried out at room temperature under open-circuit (i.e., electroless) conditions. The solution layer is confined between the metal surface and a glass plate at a constant spacing of $0.23 \mathrm{~mm}$. The copper deposits have good visual contrast against the steel which allows the recording of data with a charge-coupled device camera. For further analysis, the video signals are digitized using a PC-based frame grabber board.

* To whom correspondence should be addressed. E-mail: steinbck@ chem.fsu.edu.

10292 a J. AM. CHEM. SOC. 2002, 124, 10292-10293


Figure 1. Propagating fronts of copper deposition on the surface of a steel plate under a thin layer of cupric sulfate solution (1.07 M). Image size, 9.8 $\times 4.7 \mathrm{~cm}^{2}$; time between frames, $4 \mathrm{~s}$.

Figure 1 shows four consecutive photographs of the deposition process. In this experiment, a nearly saturated solution of cupric sulfate $(1.07 \mathrm{M})$ with a $\mathrm{pH}$ of 3.20 serves as the electrolyte. The first snapshot is taken a few seconds after the thin layer of cupric sulfate has flooded the entire surface of the passivated steel plate. It reveals two nucleation sites (upper edge of Figure 1a) that have induced nearly circular deposition fronts. The induction time between the onset of the experiment and the actual nucleation varies with the copper concentration but is typically on the order of seconds. We observed that the sample edges are the preferred location for spontaneous nucleation. In additional experiments, we found that the position of the nucleation sites can be controlled externally by removing the oxide film mechanically prior to the deposition experiment. Figure $1 b-d$ shows the subsequent evolution of the deposition fronts that eventually lead to plating of the entire sample. Under the given experimental conditions, the front velocity is $1.0 \mathrm{~mm} / \mathrm{s}$, but shows local deviations in the later stages of the deposition. These deviations induce bumpy, and sometimes blurred, front curves as seen in the right section of Figure 1d. The front has a characteristic black appearance and an estimated width of $1 \mathrm{~mm}$. In its wake, the system gradually acquires the bright, reddish color of the copper deposit. The adherence of the copper to the substrate is typically very good. Nonetheless, prolonged exposure of the plated samples to the bath generates thick, dull deposits which are easily removed from the steel and reveal an attached black oxide layer.

Figure 2a,b summarizes the front dynamics as obtained from experiments with $1.07 \mathrm{M}$ (a) and $0.27 \mathrm{M}$ (b) cupric sulfate solution at $\mathrm{pH}$ 3.20. The computer-generated overlays show the consecutive wave front positions at intervals of $0.9 \mathrm{~s}$. Consequently, the spacing between adjacent black lines increases with increasing front velocities. In the experiment of Figure $2 \mathrm{a}$, the front velocity remains constant at $1.0 \mathrm{~mm} / \mathrm{s}$ for a period of more than $20 \mathrm{~s}$ during which time a large portion of the surface has become copper plated. After 

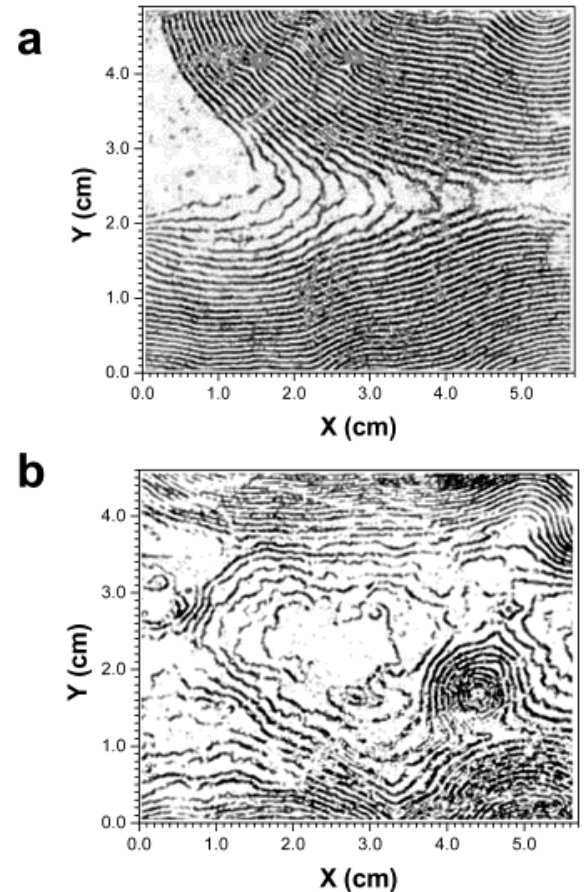

Figure 2. Additive overlay of wave front positions. Time between adjacent front curves: $0.9 \mathrm{~s}$. Initial concentration of $\mathrm{CuSO}_{4}$ : (a) $1.07 \mathrm{M}$, (b) 0.27 M. In both cases, fronts nucleate along the top and bottom edges. In (b), an additional nucleation site is located around $(x, y)=(4.4,1.8) \mathrm{cm}$.
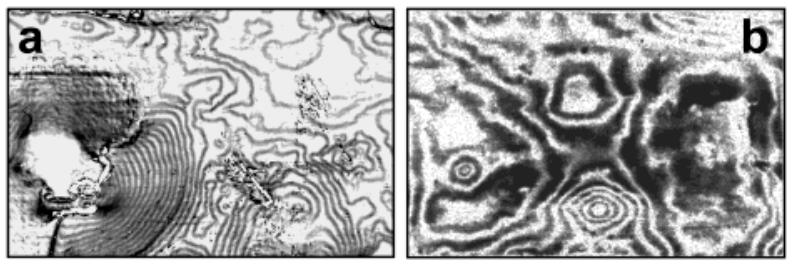

Figure 3. Additive overlay of front positions illustrating the spatio-temporal evolution observed at a constant sulfate concentration of $1.00 \mathrm{M}$ and a constant $\mathrm{pH}$ of 3.20. (a) Time between adjacent fronts $\Delta t=1.2 \mathrm{~s},\left[\mathrm{Cu}^{2+}\right]$ $=0.25 \mathrm{M}$, image size $4.9 \times 3.1 \mathrm{~cm}^{2}$; (b) $\Delta t=1.8 \mathrm{~s},\left[\mathrm{Cu}^{2+}\right]=0.025 \mathrm{M}$, image size $7.0 \times 4.6 \mathrm{~cm}^{2}$.

approximately $23 \mathrm{~s}$, however, the front accelerates and loses its smoothness as the electrodeposition zone spreads leftward in the central region of the observation area. If the initial concentration of $\mathrm{CuSO}_{4}$ is lowered to $25 \%$ (Figure 2b), the phase of constantspeed propagation shortens to about $8 \mathrm{~s}$. In this particular experiment, the subsequent plating process still occurs in a spatially distributed fashion but is only reminiscent of a self-propagating front. A further decrease in the concentration of copper sulfate leads to increasingly unstructured behavior.

For experimental conditions similar to those of Figures 1 and 2, we adjusted the $\mathrm{pH}$ of the plating bath in the range from 2-4 by addition of $\mathrm{H}_{2} \mathrm{SO}_{4}$ or $\mathrm{NaOH}$ and found no significant $\mathrm{pH}$ dependence of the front motion. Additional experiments were carried out to analyze the relative impact of cupric and sulfate ions on the spatio-temporal dynamics in this system. Figure 3 shows front overlays for experiments in which the concentration of sulfate was kept constant at $1.00 \mathrm{M}$ by addition of sodium sulfate. A comparison of Figures $2 \mathrm{~b}$ and $3 \mathrm{a}$ reveals that the addition of sodium sulfate prolongs the period of constant-speed propagation. Moreover, we find that the traveling fronts can exist at cupric ion concentrations as low as $25 \mathrm{mM}$ (Figure $3 \mathrm{~b}$ ).

Traveling fronts of copper deposition are also found for plating baths that involve copper(II) salts other than $\mathrm{CuSO}_{4}$. For example, we observed front propagation similar to Figure 1 using $1 \mathrm{M} \mathrm{Cu}$ $\left(\mathrm{NO}_{3}\right)_{2}$ solutions. These conditions require the presence of a small amount of chloride ions ${ }^{13}$ within the plating bath (e.g., $[\mathrm{NaCl}]=$ $10 \mathrm{mM}$ ) because the substrate is otherwise unsusceptible to the plating process. The addition of chloride induces the immediate nucleation of deposition fronts that propagate with velocities comparable to those observed in the case of $\mathrm{CuSO}_{4}$. However, the traveling fronts in these nitrate/chloride systems show no leading, black interface but rather the sudden appearance of the reddish copper deposit.

In conclusion, this study presents the first report of traveling fronts in the electroless copper deposition on a dissimilar metal substrate. Constant-speed fronts can exist for a period of up to 30 s. After that time, the passivation film breaks down rapidly and induces unstructured plating dynamics that affect large areas of the steel substrate in a short time. The latter behavior appears to be independent of spatial transport and/or coupling phenomena and is therefore of a phase-wavelike nature. In contrast, the initial fronts must involve an activating, short-range coupling process that accounts for their constant speed and overall dynamics. It seems likely that this coupling destabilizes or alters the passivation film in close vicinity to the front and consequently gives rise to the formation of the leading, black interface that contains copper oxide as a main constituent. The main copper deposition in the wake of the front is of minor importance for the front dynamics, which explains the insusceptibility of the velocity to changes in the cupric ion concentration. More investigations are needed to clarify the detailed reaction and coupling mechanisms that give rise to these surprising deposition fronts in one of chemistry's classic systems.

Acknowledgment. This work was supported by ACS-PRF grant no. $37278-\mathrm{G} 5$.

\section{References}

(1) Kapral, R., Showalter, K., Eds. Chemical Waves and Patterns; Kluwer: Dordrecht, Netherlands, 1995.

(2) Vanag, V. K.; Epstein, I. R. Science 2001, 294, 835-837.

(3) Bauer, G. J.; McCaskill, J. S.; Otten, H. Proc. Natl. Acad. Sci. U.S.A. 1989, 86, 7937-7941.

(4) Lechleiter, J.; Girard, S.; Peralta, E.; Clapham, D. Science 1991, 252, $123-126$

(5) Witkowski, F. X.; et al. Nature 1998, 392, 78-82.

(6) (a) Washington, R. P.; Steinbock, O. J. Am. Chem. Soc. 2001, 123, $7933-$ 7934. (b) Kobyakov, V. P.; Sharivker, S. Y.; Borovinskaya, I. P. Inorg Mater. 2002, 38, 292-295. (c) Merzhanov, A. G.; Borovinskaia, I. P.; Nersesian, M. D.; Peresada, A. G.; Morozov, I. G. Dokl. Akad. Nauk SSSR 1990, 311, 96-101. (d) Mariani, A.; Fiori, S.; Shekanov, Y.; Pojman, J. A. Macromolecules 2001, 34, 6539-6541.

(7) (a) Krischer, K.; Mazouz, N.; Grauel, P. Angew. Chem., Int. Ed. 2001 40, 850-869. (b) Agladze, K.; Thouvenel-Romans, S.; Steinbock, O. J. Phys. Chem. 2001, A105, 7356-7363. (c) Christoph, J.; Strasser, P.; Eiswirth, M.; Ertl, G. Science 1999, 284, 291-293.

(8) Matsushita, M.; Sano, M.; Hayakawa, Y.; Honjo, H.; Sawada, Y. Phys. Rev. Lett. 1984, 53, 286-289.

(9) Krastev, I.; Koper, M. T. M. Physica 1995, A213, 199-208.

(10) Hudson, J. L.; Tsotsis, T. T. Chem. Eng. Sci. 1994, 49, 1493-1572.

(11) Cowper-Coles, S. O. British Patent Number BP 25,393, November 6, 1913

(12) See, e.g.: Deckert, C. A. Plat. Surf. Finish. 1995, 82, 58-64.

(13) For a discussion of the effects of nitrate and chloride ions on the passive oxide layer of steel, see, e.g.: Bellanger, G.; Rameau, J. J. J. Mater. Sci. 2000, 35, 1759-1771.

JA026836E 